\title{
Korean Modification of the Nasal Provocation Test With House Dust Mite Antigen Following the EAACI Guidelines
}

\author{
Soo Hyun Joo (10 · Ki Jong Hyun (10) - Young Hyo Kim (i) \\ Department of Otorhinolaryngology-Head and Neck Surgery, Inha University School of Medicine, Incheon, Korea
}

Objectives. We evaluated the usefulness of the standardized nasal provocation test (NPT) protocol recently published by the European Academy of Allergy and Clinical Immunology (EAACI) and compared the utility of several parameters for diagnosing allergic rhinitis (AR) caused by house dust mites (HDM). Subjective parameters were nasal and ocular symptoms measured using a visual analog scale (VAS), and objective parameters were peak nasal inspiratory flow (PNIF), minimal cross-sectional area (MCA), and total nasal volume (TNV).

Methods. Before and after spraying Dermatophagoides pteronyssinus (DP) allergen (1,000 AU/mL, $100 \mu \mathrm{L})$ into both nostrils of 13 patients with AR (AR group) and 22 patients with non-AR (NAR group), we used VAS scores to measure nasal symptoms (nasal obstruction, rhinorrhea, sneezing, and itching) and ocular symptoms and assessed PNIF, MCA, and TNV.

Results. The AR group had significantly worse symptoms than the NAR group 15 minutes after DP challenge $(P<0.001)$. After 30 minutes, nasal obstruction and rhinorrhea remained worse in the AR group $(P<0.001)$; a similar but less marked difference was seen for sneezing $(P=0.012)$ and itching $(P=0.039)$. Ocular symptoms, PNIF, MCA, and TNV differed between groups after both 15 and 30 minutes $(P<0.05)$. The area under the receiver operating characteristic curve was higher for nasal obstruction (0.977), rhinorrhea $(0.906)$, and TNV $(0.979)$ than for sneezing $(0.755)$, itching (0.673), and MCA (0.836).

Conclusion. NPT performed according to the EAACI guidelines could help diagnose AR caused by HDM. TNV and VAS changes in nasal obstruction and rhinorrhea had higher diagnostic accuracy than other parameters.

Keywords. Nasal Provocation Tests; Rhinitis, Allergic; Rhinometry, Acoustic; Visual Analog Scale

\section{INTRODUCTION}

The nasal provocation test (NPT) directly assesses changes in a patient's symptoms and in the volume and cross-sectional area of the nasal cavity after direct administration of a suspected causative antigen into the nasal cavity [1]. The primary advan-

\footnotetext{
- Received April 8, 2020

Revised June 8, 2020

Accepted June 26, 2020

- Corresponding author: Young Hyo Kim

Department of Otorhinolaryngology-Head and Neck Surgery, Inha

University School of Medicine, 27 Inhang-ro, Jung-gu, Incheon 22332,

Korea

Tel: +82-32-890-2437, Fax: +82-32-890-3580

E-mail: inhaorl@inha.ac.kr
}

tage of the NPT over the skin prick test (SPT) is that it involves the direct stimulation and evaluation of changes in the nasal cavity, the target organ in rhinitis [2]. The NPT may therefore be used to determine which an antigen is responsible for symptoms in patients with allergic rhinitis (AR) whose SPT result is positive for multiple allergens on the SPT. The NPT can also be used to determine whether an antigen actually causes symptoms before opting for immunotherapy.The NPT also plays an important role in the diagnosis of local AR in rhinitis patients who have negative SPT results [3].

Despite these advantages, the NPT is not yet universally used for the diagnosis of AR. The most significant reason for this is that the methodology and interpretation of NPTs have not yet been standardized. As such, investigators administer antigens

Copyright @ 2021 by Korean Society of Otorhinolaryngology-Head and Neck Surgery.

This is an open-access article distributed under the terms of the Creative Commons Attribution Non-Commercial License (https://creativecommons.org/licenses/by-nc/4.0)

which permits unrestricted non-commercial use, distribution, and reproduction in any medium, provided the original work is properly cited. 
into the nasal cavity in different ways and then evaluate the resulting changes in different ways, making it difficult to objectively compare results between investigators. To address this issue, the European Academy of Allergy and Clinical Immunology (EAACI) recently published a position paper on standardization of the NPT [4]. In this paper, the EAACI described in detail the laboratory environment, the methods for allergen selection and administration, the subjective and objective parameters to be evaluated, and the criteria for positive results. However, they failed to specify the type and concentration of antigen for performing NPT. In addition, among the various criteria they proposed, it remains unknown which measurements should be evaluated in order to rapidly perform NPTs in the real-world clinical practice environment in Korea. Therefore, based on the EAACI position paper, it is necessary to develop a Korean modification of the standardized NPT protocol that is suitable for domestic clinical practice.

Therefore, in this study, we aimed to evaluate the usefulness of the standardized NPT performed according to the EAACI guidelines in clinical practice. In addition, we aimed to compare various subjective measures of symptom changes and objective parameters (peak nasal inspiratory flow [PNIF] measured by peak flowmetry, as well as the minimal cross-sectional area [MCA] and total nasal volume [TNV] of the nasal cavity measured by acoustic rhinometry) before and after antigen administration in order to identify the most useful indicators for diagnosing AR caused by house dust mites (HDM).

\section{MATERIALS AND METHODS}

\section{Subjects}

This study enrolled 35 patients (15 males and 20 females, aged 6 to 73 years, with a mean age of $38.2 \pm 19.3$ years) who visited our outpatient clinic with the symptoms of chronic rhinitis (nasal congestion, watery runny nose, and sneezing) from July 2019 to January 2020. As a diagnostic workup for allergy, we performed an SPT for all patients. The SPT was conducted using more than 40 antigens, including those from house dust mites (Dermatophagoides pteronyssinus [DP] and Dermatophagoides farina

\section{H I G H L I G G H T S}

- Nasal provocation tests (NPTs), according to the European Academy of Allergy and Clinical Immunology (EAACI) guidelines, could be highly useful for diagnosing allergic rhinitis caused by house dust mites.

- Total nasal volume and visual analog scale (VAS) changes for nasal obstruction and rhinorrhea had high diagnostic accuracy.

- We suggest a Korean modification of the EAACI NPT protocol, based on VAS scores and/or objective measurements.
[DF]), fungi, cockroaches, pollen, pets such as cats and dogs, histamine (as a positive control), and saline (as a negative control).

We excluded patients who had used antihistamines or vasoconstrictors within the last week, patients who had used intranasal steroids within the last month, and patients who had taken systemic corticosteroids within the last three months. We also excluded the following patients: those with unstable and severe systemic disease, those who had contraindications for epinephrine use, pregnant or lactating women, those who had undergone nasal surgery within the last three months, those with chronic rhinosinusitis and/or a nasal polyp confirmed by nasal endoscopy and/or imaging such as paranasal X-ray or computed tomography, and those who had been repeatedly exposed to chemical irritants or cigarette smoking. Before NPT, patients agreed to participate in this study after being provided full information from the medical staff regarding the purpose of the study. This study was approved by the Inha University Hospital Institutional Review Board Committee on Studies Involving Human Beings (IRB No. 2019-07-026).

To calculate the sample size, we first conducted a pilot study with five AR patients and five non-AR (NAR) patients with the approval of the IRB. The sample size was 35 based on the average change in symptoms in AR patients $(16.3 \mathrm{~mm})$, average change in symptoms in NAR patients $(3.5 \mathrm{~mm})$, standard deviation $(10.5 \mathrm{~mm})$, alpha error (0.5), and statistical power of $95 \%$.

We classified the 35 patients as follows according to their SPT results: group A (AR group: $n=13$, patients with strongly positive results for DP/DF and negative results for all the other antigens) and group B (NAR group: $n=22$, patients negative for all antigens tested including DP and DF). We defined "strongly positive" as "when the size of the skin wheal caused by an antigen is equal to or greater than the size of the wheal caused by histamine" and "negative" as "when the wheal size is equal to that of saline or there is no wheal." The demographic characteristics of each group are summarized in Table 1.

Standardized NPT method based on the EAACI position paper Laboratory setting and acclimatization

We sought to faithfully follow the recommendations of the EAA-

Table 1. Demographic variables and ARIA classification of symptom severity in 35 patients divided into AR and NAR groups

\begin{tabular}{lccc}
\hline Variable & $\begin{array}{c}\text { AR group } \\
(n=13)\end{array}$ & $\begin{array}{c}\text { NAR group } \\
(n=22)\end{array}$ & $P$-value ${ }^{\text {a) }}$ \\
\hline Sex (male:female) & $7: 6$ & $8: 14$ & NS \\
Age (yr), mean \pm SD & $27.3 \pm 11.8$ & $44.7 \pm 20.2$ & 0.013 \\
ARIA classification & & & \\
$\quad$ Intermittent:persistent & $4: 9$ & $10: 12$ & NS \\
$\quad$ Mild:moderate-severe & $10: 3$ & $12: 10$ & NS \\
\hline
\end{tabular}

ARIA, Allergic Rhinitis and its Impact on Asthma; AR, allergic rhinitis; NAR, non-allergic rhinitis; NS, not significant; SD, standard deviation.

${ }^{a)}$ Chi-square test. 
CI position paper when performing the NPT for all patients [4]. The laboratory environment was controlled to maintain a constant temperature $\left(20^{\circ} \mathrm{C} \pm 1.5^{\circ} \mathrm{C}\right)$ and humidity $(40 \%-60 \%)$. The patients were allowed to acclimatize to these conditions by waiting for at least 15 minutes before the test.

\section{Antigen and sprayer for provocation}

When selecting the test antigens, we compared the concentrations of antigens used by several researchers and opted to use antigens that are readily commercially available in many countries, including the Republic of Korea. As a result, we purchased a standardized DP 10,000 AU/mL solution (\#6692; HollisterStier Allergy, Spokane, WA, USA) and diluted it 1:10 with physiological saline before using it for the NPT. As a control challenge (to rule out non-specific hyper-reactivity), we used physiological saline. Using a metered dose pump sprayer that dispensed a constant dose each time, we sprayed $100 \mu \mathrm{L}$ of physiological saline or DP solution into each nostril of the patient.

\section{Subjective and objective measurements}

To measure subjective symptoms, we used the visual analog scale (VAS) to assess nasal obstruction, rhinorrhea, sneezing, nasal pruritis, and ocular symptoms as recommended in the EAACI position paper [4]. We measured symptoms on a scale from $0 \mathrm{~mm}$ (no symptoms) to $100 \mathrm{~mm}$ (the worst possible symptoms) using a standardized $100 \mathrm{~mm}$ VAS ruler.

To obtain an objective measure of PNIF, we used a portable inspiratory flow meter (Clement Clarke International, Harlow, UK). We applied the mask connected to the flow meter to the patients' nose and mouth and asked the patients to close their mouth and inhale as much as possible through their nose. To obtain objective measurements of MCA and TNV, we used an A1 Acoustic Rhinometer (GM Instrument, Irvine, UK). We defined the smallest cross-sectional area of the nasal cavity as the MCA, and defined the sum of the cross-sectional areas from $2 \mathrm{~cm}$ away from the nostril to $5 \mathrm{~cm}$ away from the nostril as the TNV.

\section{NPT protocol}

As a "baseline measurement," we measured subjective symptoms (VAS) and objective indicators (PNIF, MCA, and TNV) before patients were presented with a challenge. After that, we applied the control solution $(100 \mu \mathrm{L}$ physiological saline) to each nostril of the patient. Ten minutes after the control challenge, we repeated measurements for VAS, PNIF, MCA, andTNV.

We defined "VAS change" as [(post-challenge VAS)-(baseline VAS)]. For objective measurements including PNIF, MCA, and TNV, we defined \% change as:

$$
\frac{[(\text { baseline value })-(\text { post }- \text { challenge value })]}{\text { Baseline value }} \times 100(\%) .
$$

If the VAS change after the control challenge was more than $27.5 \mathrm{~mm}$, or if the change in any objective measure was greater than $20 \%$ of the baseline value, we concluded that the patient had non-specific hyper-reactivity and discontinued examination. We also followed EAACI recommendations regarding these non-specific hyper-reactivity criteria. In our study, none of the 35 patients had nonspecific hyper-reactivity. We then sprayed $100 \mu \mathrm{L}$ of DP solution into both sides of the patients' nasal cavity. Following this, we repeated the measurement of VAS for nasal and ocular symptoms, PNIF using the flow meter, and MCA and TNV using the acoustic rhinometer. We then calculated the VAS change and the \% change values for the objective indicators based on the above formula.

\section{Statistical analysis}

We used the $\mathrm{F}$ test to compare variances and the unpaired $t$-test withWelch's correction to compareVAS changes and the \% change in PNIF, MCA, and TNV between the AR and NAR groups. To determine which measure (of the VAS changes for each symptom and the \% change values for the objective indicators) was most useful in the diagnosis of AR, we conducted receiver operating characteristic (ROC) curve analysis. We used Prism 5.0 and IBM SPSS ver. 22.0 (IBM Corp., Armonk, NY, USA) for statistical analyses and considered a $P$-value of less than 0.05 to be statistically significant.

\section{RESULTS}

\section{Comparison of subjective symptom changes after the DP challenge}

After the control challenge with physiological saline, the AR and NAR groups showed no significant difference in any nasal symptoms ( $P>0.05$ for all symptoms). After administration of the DP antigen, the AR group showed a significant worsening of nasal obstruction compared to the NAR group at both 15 minutes (46.5 \pm $6.1 \mathrm{~mm}$ vs. $-1.3 \pm 2.6 \mathrm{~mm}, P<0.001)$ and 30 minutes $(42.7 \pm$ $5.5 \mathrm{~mm}$ vs. $-4.0 \pm 3.1 \mathrm{~mm}, P<0.001)$. Rhinorrhea was also significantly worse in the AR group than in the NAR group, at both 15 minutes $(66.2 \pm 8.2 \mathrm{~mm}$ vs. $-3.2 \pm 4.2 \mathrm{~mm}, P<0.001)$ and 30 minutes ( $48.5 \pm 8.9 \mathrm{~mm}$ vs. $-6.4 \pm 4.4 \mathrm{~mm}, P<0.001$ ) after administration of the DP antigen (Fig. 1A and B).

Regarding sneezing and itching, the AR group showed significantly worse symptoms than the NAR group at 15 minutes after the DP challenge (for sneezing: $75.4 \pm 4.6 \mathrm{~mm}$ vs. $-6.8 \pm 3.1 \mathrm{~mm}$, $P<0.001$; for itching: $56.2 \pm 8.2 \mathrm{~mm}$ vs. $-2.5 \pm 4.0 \mathrm{~mm}, P<$ $0.001)$. Although the difference between the groups was still statistically significant 30 minutes after DP administration, it was less marked than at 15 minutes (for sneezing: $13.9 \pm 6.8 \mathrm{~mm}$ vs. $-7.3 \pm 3.3 \mathrm{~mm}, P=0.012$; for itching: $15.4 \pm 9.1 \mathrm{~mm}$ vs. $-6.6 \pm$ $3.5 \mathrm{~mm}, P=0.039$ ) (Fig. $1 \mathrm{C}$ and D).

Both 15 and 30 minutes after antigen administration, ocular symptoms in the AR group were significantly worse than those in the NAR group, but this difference was smaller than that for 

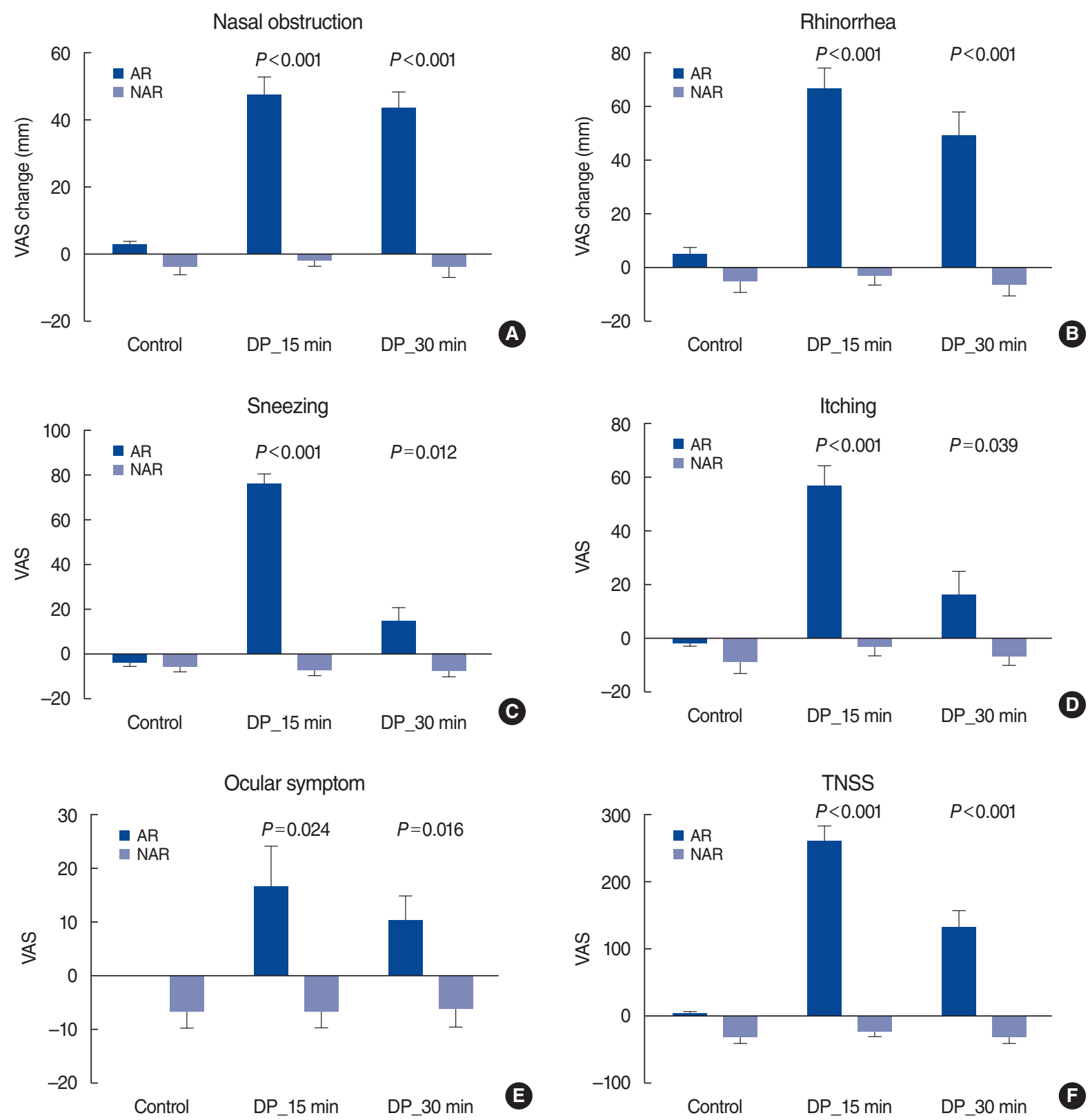

Fig. 1. Changes in symptoms measured with a visual analog scale (VAS) before and after a nasal challenge with Dermatophagoides pteronyssinus (DP) allergen extract. (A) Nasal obstruction. (B) Rhinorrhea. (C) Sneezing. (D) Itching. (E) Ocular symptom. (F) Total nasal symptom score (TNSS). VAS change: (post-challenge VAS)-(baseline VAS) of each symptom. DP_15 min, 15 minutes after the DP challenge; DP_30 min, 30 minutes after the DP challenge; AR, allergic rhinitis; NAR, non-allergic rhinitis. Unpaired t-test with Welch's correction, statistical significance: $P<0.05$.

the other nasal symptoms $(P=0.024$ and $P=0.016$ at 15 and 30 minutes, respectively). When the sum of all symptoms was defined by the total nasal symptom score (TNSS), there was a significant difference between the AR and NAR groups at both 15 minutes $(260.4 \pm 23.2 \mathrm{~mm}$ vs. $-20.1 \pm 11.1 \mathrm{~mm}, P<0.001)$ and 30 minutes $(130.4 \pm 27.5 \mathrm{~mm}$ vs. $-30.1 \pm 11.4 \mathrm{~mm}, P<0.001)$ after the DP challenge (Fig. 1E and F).
Comparison of changes in objective measures including PNIF, MCA, and TNV after the DP challenge

The AR group showed significantly lower PNIF than the NAR group at both 15 minutes $(40.8 \% \pm 10.6 \%$ vs. $5.1 \% \pm 4.3 \%, P=$ $0.007)$ and 30 minutes $(40.4 \% \pm 12.3 \%$ vs. $11.1 \% \pm 69.3 \%, P=$ 0.010 ) after DP administration. MCA was also significantly lower in the AR group than in the NAR group at both 15 minutes $(50.6 \% \pm 9.9 \%$ vs. $17.6 \% \pm 4.1 \%, P=0.007)$ and 30 minutes $(56.2 \% \pm 8.2 \%$ vs. $18.0 \% \pm 4.3 \%, P<0.001)$. This trend was also 

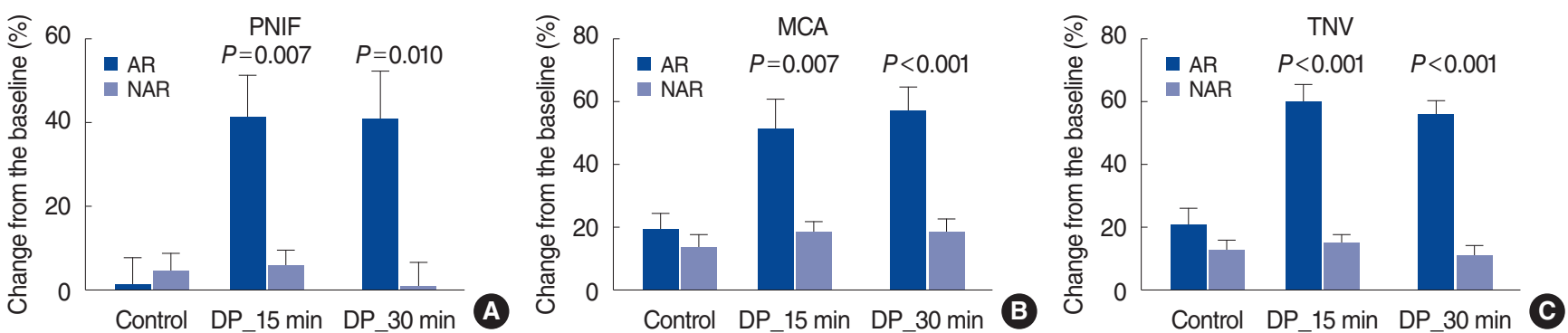

Fig. 2. \%Change in peak nasal inspiratory flow (PNIF; A), minimal cross-sectional area (MCA; B), and total nasal volume (TNV; C) before and after a nasal challenge with Dermatophagoides pteronyssinus (DP) allergen extract. DP_15 min, 15 minutes after the DP challenge; DP_30 min, 30 minutes after the DP challenge; AR, allergic rhinitis; NAR, non-allergic rhinitis. Unpaired t-test with Welch's correction, statistical significance: $P<0.05$.
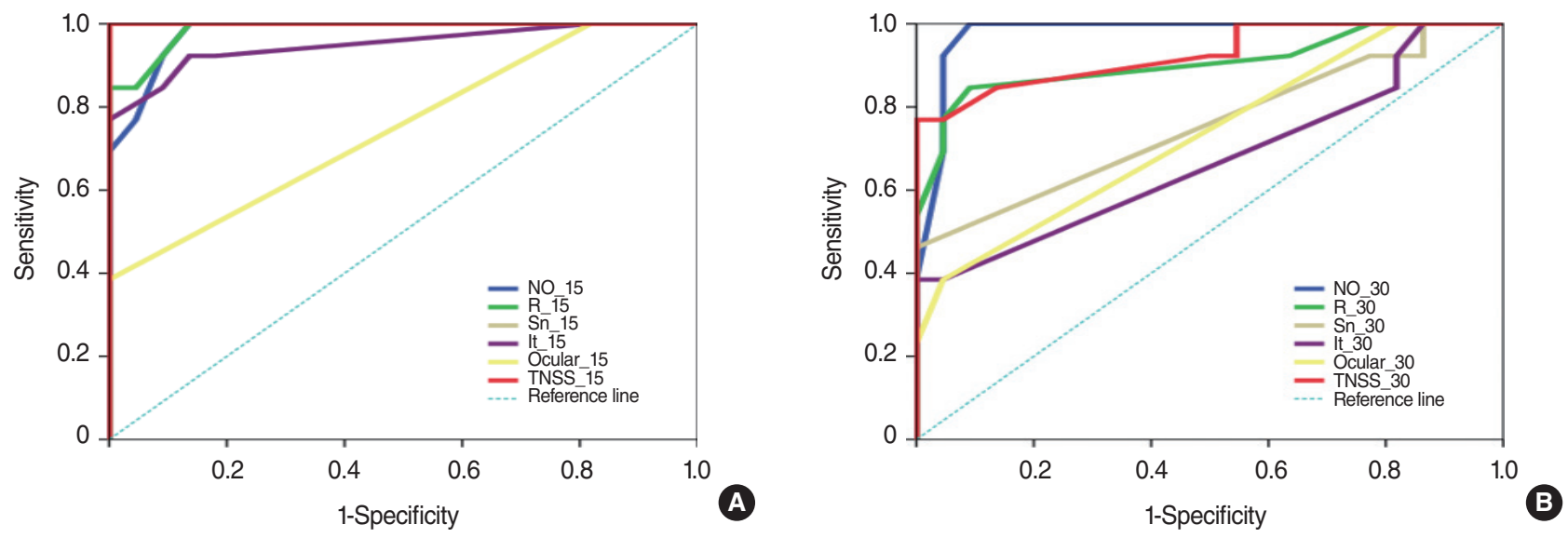

Fig. 3. Receiver operating characteristic curve analysis for changes in symptoms before and after a nasal challenge with Dermatophagoides pteronyssinus (DP) allergen extract. (A) After 15 minutes. (B) After 30 minutes. NO, nasal obstruction; R, rhinorrhea; Sn, sneezing; It, itching; Ocular, ocular symptoms; TNSS, total nasal symptom score; _15, 15 minutes after the DP challenge;_30, 30 minutes after the DP challenge.

true forTNV (after 15 minutes: $59.7 \% \pm 6.0 \%$ vs. $14.5 \% \pm 3.0 \%$, $P<0.001$; after 30 minutes: $55.7 \% \pm 4.8 \%$ vs. $10.3 \% \pm 3.4 \%$, $P<0.001$ ) (Fig. 2).

\section{ROC curve analysis for subjective symptoms}

The ROC curve analysis for changes in each symptom at 15 minutes after the DP challenge showed that all nasal symptoms (nasal obstruction, rhinorrhea, sneezing, and itching) had very high area under the curve (AUC) values of 0.94 or more (all $P<0.001$ ). In contrast, ocular symptoms had an AUC of $0.748(P=0.015)$, making them of somewhat less diagnostic value than the other symptoms.

At 30 minutes after the DP challenge, the AUC values remained quite high for nasal obstruction $(0.977, P<0.001)$ and rhinorrhea $(0.906, P<0.001)$. In contrast, the AUC values were lower, indicating less diagnostic power, for sneezing $(0.755, P=0.013)$, itching $(0.673, P=0.091)$, and ocular symptoms $(0.731, P=0.024)$. The AUC of TNSS was $1.000(P<0.001)$ and $0.927(P<0.001)$ at 15 and 30 minutes, respectively (Fig. 3). According to the results of the ROC analysis, we set cutoff values for each subjective symptom parameter. The cutoff value, sensitivity, specificity,
Table 2. The cutoff value, sensitivity, specificity, recall (true positive rate), and precision (positive predictive value) for subjective symptom parameters

\begin{tabular}{|c|c|c|c|c|c|}
\hline Variable & $\begin{array}{l}\text { Cutoff } \\
\text { value } \\
(\mathrm{mm})\end{array}$ & Sensitivity & Specificity & $\begin{array}{l}\text { Recall } \\
\text { (true } \\
\text { positive } \\
\text { rate) }\end{array}$ & $\begin{array}{c}\text { Precision } \\
\text { (positive } \\
\text { predictive } \\
\text { value) }\end{array}$ \\
\hline \multicolumn{6}{|c|}{ DP_15 min VAS change of } \\
\hline Nasal obstruction & 15.0 & 92.3 & 90.9 & 92.3 & 85.7 \\
\hline Rhinorrhea & 15.0 & 100.0 & 86.4 & 100.0 & 81.3 \\
\hline Sneezing & 20.0 & 100.0 & 100.0 & 100.0 & 100.0 \\
\hline Itching & 25.0 & 84.6 & 90.9 & 84.6 & 84.6 \\
\hline Ocular symptom & 5.0 & 38.5 & 100.0 & 38.5 & 100.0 \\
\hline TNSS & 100.0 & 100.0 & 100.0 & 100.0 & 100.0 \\
\hline \multicolumn{6}{|c|}{ DP_30 min VAS change of } \\
\hline Nasal obstruction & 15.0 & 92.3 & 95.5 & 92.3 & 92.3 \\
\hline Rhinorrhea & 5.0 & 84.6 & 90.9 & 84.6 & 84.6 \\
\hline Sneezing & 10.0 & 46.2 & 100.0 & 46.2 & 100.0 \\
\hline Itching & 7.5 & 38.5 & 100.0 & 38.5 & 100.0 \\
\hline Ocular symptom & 5.0 & 38.5 & 95.5 & 38.5 & 83.3 \\
\hline TNSS & 80.0 & 76.9 & 100.0 & 100.0 & 100.0 \\
\hline
\end{tabular}

DP, Dermatophagoides pteronyssinus; DP_15 min, 15 minutes after the DP challenge; VAS, visual analog scale; TNSS, total nasal symptom score; DP_30 min, 30 minutes after the DP challenge. 

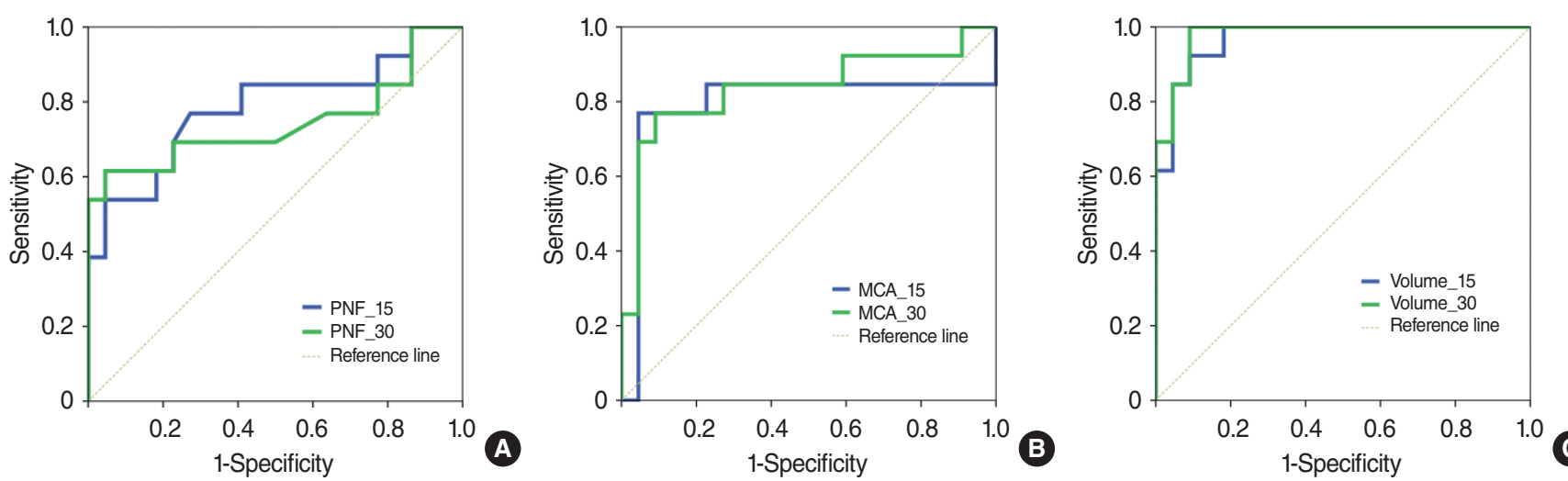

Fig. 4. Receiver operating characteristic curve analysis for the percent change in peak nasal inspiratory flow (PNIF; A), minimal cross-sectional area (MCA; B), and total nasal volume (TNV; C) before and after a nasal challenge with Dermatophagoides pteronyssinus (DP) allergen extract. _15, 15 minutes after the DP challenge; _30, 30 minutes after the DP challenge.

Table 3. The cutoff value, sensitivity, specificity, recall (true positive rate), and precision (positive predictive value) for objective parameters

\begin{tabular}{|c|c|c|c|c|c|}
\hline Variable & $\begin{array}{c}\text { Cutoff } \\
\text { value (\%) }\end{array}$ & Sensitivity & Specificity & $\begin{array}{l}\text { Recall (true } \\
\text { positive } \\
\text { rate) }\end{array}$ & $\begin{array}{c}\text { Precision } \\
\text { (positive } \\
\text { predictive } \\
\text { value) }\end{array}$ \\
\hline \multicolumn{6}{|c|}{ DP_15 min \%change of } \\
\hline PNIF & 16.1 & 76.9 & 72.7 & 76.9 & 62.5 \\
\hline TNV & 32.3 & 92.3 & 90.9 & 92.3 & 85.7 \\
\hline MCA & 22.9 & 84.6 & 77.3 & 84.6 & 68.8 \\
\hline \multicolumn{6}{|c|}{ DP_30 min \%change of } \\
\hline PNIF & 15.5 & 69.2 & 77.3 & 69.2 & 64.3 \\
\hline TNV & 26.8 & 100.0 & 90.9 & 100.0 & 86.7 \\
\hline MCA & 41.4 & 76.9 & 90.9 & 76.9 & 83.3 \\
\hline
\end{tabular}

DP, Dermatophagoides pteronyssinus; DP_15 min, 15 minutes after the DP challenge; PNIF, peak nasal inspiratory flow; TNV, total nasal volume; MCA, minimal cross-sectional area; DP_30 min, 30 minutes after the DP challenge.

recall (true positive rate) and precision (positive predictive value) are summarized in Table 2.

\section{ROC curve analysis for objective measurements}

At 15 and 30 minutes after the DP challenge, the AUC of PNIF changes was $0.785(P=0.005)$ and $0.743(P=0.018)$, respectively. Compared to the AUC of MCA (AUC $=0.794, P=0.004$ after 15 minutes; $\mathrm{AUC}=0.836, P=0.001$ after 30 minutes), the AUC of TNV was consistently higher (AUC $=0.969, P<0.001$ after 15 minutes, AUC $=0.979, P<0.001$ after 30 minutes) (Fig. 4). According to the results of the ROC analysis, we set cutoff values for each objective parameter. The cutoff value, sensitivity, specificity, recall (true positive rate) and precision (positive predictive value) are summarized in Table 3.

\section{DISCUSSION}

The NPT has the advantages of directly stimulating the nose, which is the target organ in AR, and of involving direct observations of changes in the nasal cavity [5]. Nevertheless, since there is no consensus on the details of the NPT method among researchers, the test has been performed in many different ways. The EAACI recently published a position paper on the NPT, providing detailed recommendations on its implementation [4]. Our study is significant because it is the first to implement a standardized NPT protocol following the guidelines in the EAACI position paper and to evaluate the results and usefulness of the NPT.

EAACI recommends the use of instruments to measure subjective symptoms and objective changes in the nasal cavity for the NPT. Accordingly, we evaluated changes in five symptoms using VAS scores: nasal obstruction, rhinorrhea, sneezing, itching, and ocular symptoms. At the same time, PNIF, MCA, and TNV were measured using peak flowmetry and acoustic rhinometry to obtain objective measurements of these parameters. The percent change in these measurements before and after provocation was calculated. The measurement of both VAS changes and percent changes are recommended in the EAACI position paper [4].

After the DP challenge, we found that all nasal symptoms and ocular symptoms worsened significantly in the AR group compared to the NAR group. These results are consistent with previous studies. In our previous study of 208 AR patients and 222 NAR patients, the AR group had significantly worse nasal symptoms after a DP challenge than the NAR group [6]. However, the DP solution (Allergopharma, Darmstadt, Germany) used in previous studies has been discontinued and can no longer be used. Our previous study has a further disadvantage in that it was performed before the publication of the EAACI position paper regarding NPT standardization. Thus, the current study is the first to demonstrate that the standardized NPT protocol yields results 
consistent with previous studies. Interestingly, there was a marked difference between the AR and NAR groups in terms of nasal obstruction and rhinorrhea symptoms up to 30 minutes after DP administration $(P<0.001)$. With regard to sneezing and itching symptoms, the difference between the two groups diminished by 30 minutes after DP administration (although a significant difference still remained). The VAS score change in the AR group was $13.9 \pm 6.8 \mathrm{~mm}$ for sneezing and $15.4 \pm 9.1 \mathrm{~mm}$ for itching at 30 minutes after DP administration. These values are less than the criteria for "clearly positive $(55 \mathrm{~mm})$ " and "moderately positive $(23 \mathrm{~mm})$ " presented in the EAACI position paper [4]. Therefore, the present study found that VAS score changes for nasal obstruction and rhinorrhea may be more effective than VAS score changes for sneezing and itching in distinguishing between AR and NAR 30 minutes after DP administration.

The VAS score change in ocular symptoms was significantly higher in the AR group at 15 minutes and 30 minutes after DP administration. However, theVAS score change was $16.2 \pm 8.3 \mathrm{~mm}$ after 15 minutes and $10.0 \pm 4.9 \mathrm{~mm}$ after 30 minutes; these values are lower than the criteria in the EAACI guidelines. These findings are in line with previous reports. According to Tomljenovic et al. [7], the VAS score change for eye itching sensation was approximately 1.7 and that in tear production was approximately 2.6 after an allergen challenge. Therefore, we propose using changes in the VAS scores for nasal symptoms, rather than ocular symptoms, after the NPT.

In addition to subjectively assessing changes in symptoms, we measured objective parameters such as PNIF, MCA, and TNV. We found that all these parameters were significantly lower in the AR group than in the NAR group at 15 and 30 minutes after DP administration. These results are also consistent with previous findings. In addition to our studies using acoustic rhinometry for NPT [2,8-11], Wandalsen et al. [12] also found that acoustic rhinometry was an effective measure for NPT in adolescents and children. The current study is the first to perform the NPT according to the standardized protocol in the EAACI position paper and to measure the changes in these objective parameters.

We performed ROC curve analysis to determine which of the subjective and objective parameters had the highest sensitivity and specificity for AR diagnosis. The VAS scores for all nasal symptoms (nasal obstruction, rhinorrhea, sneezing, and itching) at 15 minutes after DP exposure had AUC values of over 0.94. Ocular symptoms, in contrast, had a lower AUC (0.748). These findings can provide valuable information to aid physicians implementing NPTs at their outpatient clinics. For many doctors who would like to perform NPTs, time constraints are a major obstacle. If there is a need to wait 30 minutes after DP exposure, the total test time would be 40 minutes or more. In this study, we propose that results with reliable sensitivity and specificity could be obtained 15 minutes after DP exposure, thus providing useful information for busy doctors. The ROC curve analysis of the results at 30 minutes after DP exposure further supports the use of the NPT at 15 minutes. While nasal obstruction and rhinorrhea still had high AUC values even after 30 minutes, the remaining symptoms (sneezing, itching, and ocular symptoms) had significantly lower AUC values, which reduces their usefulness as diagnostic criteria.

Our research, unlike previous studies, used several instruments such as peak flowmetry and acoustic rhinometry to measure objective indicators such as PNIF, MCA, and TNV, enabling an evaluation and comparison of their usefulness. The most notable finding in this study was that the AUC of PNIF was lower than that of subjective symptoms. It is very easy to assume that objective measurements are more reliable than subjective symptoms, but our findings suggest this might not be true. According to Boelke et al. [13], PNIF decreased by an average of approximately $31.7 \%$ at 30 minutes after the DP challenge. Of course, it is difficult to make direct comparisons between their study and ours because of the different concentrations of antigen used, but these values are lower than the " $40 \%$ reduction from the baseline" recommended by the EAACI guidelines. We found that TNV had a higher AUC value than PNIF or MCA. Therefore, we suggest that it could be most useful to measure TNV using acoustic rhinometry as part of the NPT.

One of the drawbacks of this study is the relatively small number of patients included. Patient numbers were limited because it took considerable time to measure several subjective and objective parameters for the standardized NPT in each patient. Another limitation of this study is that the control group consisted of NAR patients, not healthy volunteers. This issue has already been discussed in detail in our previous papers. Healthy volunteers usually have no symptoms, while NAR patients have some degree of rhinitis symptoms. Therefore, it can be assumed that the difference in symptoms between a healthy volunteer and an AR patient would be much larger than that between a NAR patient and an AR patient. In addition, our results are especially useful in the context of clinical practice because it is more common to discriminate between AR and NAR in patients with rhinitis symptoms.

We used the DP antigen for the NPT in this study. This was because DP is the most common causative antigen for AR in the Republic of Korea, the country in which we conducted the study. Diverse kinds of antigen solutions have been commercially developed for subcutaneous immunotherapy. Therefore, when researchers from various countries select different antigens and perform the standardized NPT according to the EAACI position paper, we could compare their results to develop further meaningful conclusions.

In conclusion, this study is the first to suggest a Korean modification of the standardized NPT using HDM antigen extracts based on the EAACI position paper and to demonstrate the usefulness of this protocol. In addition, changes in nasal symptoms and in various objective indicators (e.g., PNIF, TNV, and MCA) 
after the DP challenge proved to be very useful for the diagnosis of AR caused by HDM.

\section{CONFLICT OF INTEREST}

No potential conflict of interest relevant to this article was reported.

\section{ACKNOWLEDGMENTS}

This study was supported by the Basic Science Research Program through the National Research Foundation of Korea (NRF2020R1F1A1064194) and Inha University Research Grant.

\section{ORCID}

Soo Hyun Joo https://orcid.org/0000-0002-2328-5825

Ki Jong Hyun https://orcid.org/0000-0001-8905-4053

Young Hyo Kim https://orcid.org/0000-0002-3623-1770

\section{AUTHOR CONTRIBUTIONS}

Conceptualization: YHK. Data curation: KJH. Formal analysis: SHJ. Funding acquisition: YHK. Methodology: SHJ, KJH. Project administration: SHJ, YHK. Visualization: KJH,YHK. Writingoriginal draft: SHJ, KJH.Writing-review \& editing: YHK.

\section{REFERENCES}

1. Agache I, Bilo M, Braunstahl GJ, Delgado L, Demoly P, Eigenmann P, et al. In vivo diagnosis of allergic diseases--allergen provocation tests. Allergy. 2015 Apr;70(4):355-65.

2. Jang TY, Kim YH. Nasal provocation test is useful for discriminating allergic, nonallergic, and local allergic rhinitis. Am J Rhinol Allergy. 2015 Jul-Aug;29(4):e100-4.

3. Rondon C, Campo P, Herrera R, Blanca-Lopez N, Melendez L, Canto $\mathrm{G}$, et al. Nasal allergen provocation test with multiple aeroallergens detects polysensitization in local allergic rhinitis. J Allergy Clin Immunol. 2011 Dec;128(6):1192-7.

4. Auge J,Vent J,Agache I, Airaksinen L, Campo Mozo P, Chaker A, et al. EAACI Position paper on the standardization of nasal allergen challenges. Allergy. 2018 Aug;73(8):1597-1608.

5. Kim KS, Jang TY, Kim YH. Usefulness of Allerkin house dust mite extract for nasal provocation testing. Clin Exp Otorhinolaryngol. 2017 Sep;10(3):254-8.

6. Kim YH, Jang TY. Proposed diagnostic standard using visual analogue scale and acoustic rhinometry in nasal provocation test in allergic patients. Auris Nasus Larynx. 2011 Jun;38(3):340-6.

7. Tomljenovic D, Baudoin T, Megla ZB, Vagic D, Hellings P, Kalogjera L. Nasal and ocular responses after specific and nonspecific nasal challenges in seasonal allergic rhinitis. Ann Allergy Asthma Immunol. 2016 Mar;116(3):199-205.

8. Park KI, Jang TY, Yang SC, Hong HS, Kim YH. Correlation of nasal eosinophilia and response after nasal provocation test in patients with nonallergic rhinitis. Otolaryngol Head Neck Surg. 2018 Aug; 159(2):231-7.

9. Chang GU, Jang TY, Kim KS, Choi H, Kim YH. Nonspecific hyperreactivity and localized allergy: cause of discrepancy between skin prick and nasal provocation test. Otolaryngol Head Neck Surg. 2014 Feb;150(2):194-200.

10. Kim YH, Jang TY. Nasal provocation test using allergen extract versus cold dry air provocation test: which and when? Am J Rhinol Allergy. 2013 Mar-Apr;27(2):113-7.

11. Kim YH, Yang TY, Lee DY, Ko KJ, Shin SH, Jang TY. Evaluation of acoustic rhinometry in a nasal provocation test with allergic rhinitis. Otolaryngol Head Neck Surg. 2008 Jul;139(1):120-3.

12. Wandalsen GF, Mendes AI, Matsumoto F, Sole D. Acoustic rhinometry in nasal provocation tests in children and adolescents. J Investig Allergol Clin Immunol. 2016;26(3):156-60.

13. Boelke G, Berger U, Bergmann KC, Bindslev-Jensen C, Bousquet J, Gildemeister J, et al. Peak nasal inspiratory flow as outcome for provocation studies in allergen exposure chambers: a GA2 LEN study. Clin Transl Allergy. 2017 Sep;7:33. 